\title{
Separating stretching from folding in fluid mixing
}

\author{
Douglas H. Kelley and Nicholas T. Ouellette ${ }^{\star}$
}

Fluid mixing controls many natural and industrial processes, including the spread of air pollution ${ }^{1}$, mass transfer and reactions in microfluidic devices ${ }^{2,3}$ and the detection of odours or other chemical signals ${ }^{4}$. Strongly nonlinear flows enhance mixing by chaotic advection ${ }^{5,6}$, stretching and folding ${ }^{7,8}$ fluid volumes. Though these processes have been studied in simple models ${ }^{9,10}$, stretching and folding are difficult to distinguish in real flows with complex spatiotemporal structure. Here we report measurements of these two distinct processes in a two-dimensional laboratory flow. We decouple stretching and folding using tools developed for analysing glassy solids ${ }^{11}$ and colloids ${ }^{12}$, breaking fluid deformation into a linear, affine component (primarily stretching) and a nonlinear, non-affine component (primarily folding). Short-time deformation is dominated by stretching, whereas folding occurs only after fluid elements are elongated. The relative strength of the two processes depends strongly on space and time; foldingdominated regions are initially isolated, but later grow to fill space.

Mixing is fundamentally a diffusion process: at the boundary of an impurity in a fluid, the concentration gradient is large and material flows until the gradient vanishes. Diffusion alone is inefficient for large-scale transport, such as is required in industrial mixers or observed in geophysical flow. Moving fluids, however, can greatly enhance mixing through chaotic advection ${ }^{5,6}$. As the fluid moves, the region containing the impurity is strongly deformed, the length of its boundary grows exponentially and diffusion becomes efficient. The key to understanding chaotic mixing, then, is the characterization of the deformation of fluid elements ${ }^{8}$. As first described by Reynolds ${ }^{7}$, this process is one of stretching, which increases the length of the interface, and folding, which constrains the fluid element to fill a finite region of space. These geometric processes are often studied in simple mathematical models such as the baker's or horseshoe maps ${ }^{9,10}$. Such models, however, differ from actual fluid flow in that they are discrete, periodic and highly idealized. Although stretching and folding have been described qualitatively in real flows ${ }^{2,13,14}$, they have not been quantitatively distinguished spatially, temporally or dynamically in flows with complex spatiotemporal structure.

Deformation of a material volume consists of the relative motion, and potentially rearrangement, of infinitesimal fluid elements. These relative displacements can be broadly characterized as either affine-that is, some combination of rotation, shear, dilation or compression $^{15}$ - or non-affine. Affine deformation is linear, and can be represented by a matrix operator. Non-affine deformation is nonlinear, and generally consists of irreversible rearrangements of the constituent volume elements ${ }^{11}$. This distinction between affine and non-affine deformation has been used to study shear transformation zones and plasticity in metallic glasses ${ }^{11,16}$, flow in granular systems ${ }^{17}$ and glassy colloidal suspensions ${ }^{12}$. As stretching is affine and folding is non-affine, we can characterize the chaotic mixing process in a fluid using this same distinction.

We study the deformation of fluid elements in a nearly twodimensional experimental fluid flow that is chaotic in both space and time. We drive flow in a thin layer of salt water $(16 \%$ by mass $\mathrm{NaCl}$ ), with a lateral area of $86 \times 86 \mathrm{~cm}^{2}$ and a depth of $4 \mathrm{~mm}$. A square lattice of magnets of alternating polarity lies below the fluid. When we run d.c. electric current (up to $1 \mathrm{~A}$ ) through the salt water, Lorentz forces produce motion that lies almost entirely in the plane $\mathrm{e}^{13,18-21}$. We measure the fluid motion by tracking ${ }^{22}$ $51 \mu \mathrm{m}$ polystyrene particles using a 4 megapixel digital camera at 60 frames per second. The particles are small enough to follow the flow accurately ${ }^{21}$ and lie at the interface between the salt water and a less dense pure water layer, eliminating surface-tension-driven interactions between the particles ${ }^{23}$. We image only the central $31 \times 23 \mathrm{~cm}^{2}$ of the flow, so that boundary effects are negligible, and track up to 35,000 particles per frame. We reconstruct the full velocity field by projecting the measurements onto a basis of streamfunction eigenmodes ${ }^{24,25}$. As the velocity field is well resolved in space and time, we can construct the trajectories of arbitrarily located fluid elements by numerically integrating their equations of motion through the measured fields ${ }^{18,19,21}$. We define the Reynolds number $\mathrm{Re}=U L / v$ on the basis of the root-mean-square velocity $U$, the magnet spacing $L=2.54 \mathrm{~cm}$ and the kinematic viscosity $v=0.0124 \mathrm{~cm}^{2} \mathrm{~s}^{-1}$. Dimensional arguments give the characteristic forcing timescale as $T_{L}=L / U$. At low Re, the flow is a vortex lattice; as $\operatorname{Re}$ increases past a critical value $\operatorname{Re}_{\mathrm{c}} \sim 75$, the symmetries of the forcing are broken and the flow becomes chaotic in space and time ${ }^{20}$. A sample velocity field at $\mathrm{Re}=185$ (well above the transition to chaos) with $T_{L}=2.8 \mathrm{~s}$ is shown in Fig. 1a.

To study deformation quantitatively, we need mathematical measures of the affine and non-affine deformation experienced by the fluid. Working in the Lagrangian framework, where the fluid is represented by a continuum of fluid elements, we parameterize material volumes by a central point $\mathbf{x}^{(0)}(t)$ and a cluster of $N$ neighbours $\mathbf{x}^{(n)}(t)$ equally spaced on a circle of radius $d_{0}$ around the central point, where $n$ indexes the elements of the cluster. The relative positions of the neighbours are given by $\mathbf{d}^{(n)}(t)=$ $\mathbf{x}^{(n)}(t)-\mathbf{x}^{(0)}(t)$, where $\left|\mathbf{d}^{(n)}(t)\right|=d_{0}$. After a time $\tau_{\mathrm{d}}$, which we call the deformation time, the vectors describing the new shape of the cluster are $\mathbf{d}^{(n)}\left(t+\tau_{\mathrm{d}}\right)$. By fitting the deformation of the cluster to a linear (affine) model, we extract both the affine and non-affine parts of the deformation separately. More precisely, we compute the non-affine (folding) deformation of the cluster as

$$
\begin{aligned}
D^{2}\left(\mathbf{x}, t, \tau_{\mathrm{d}}\right)= & \min _{\alpha_{i j}}\left[\frac { 1 } { d _ { 0 } ^ { 2 } N } \sum _ { n = 1 } ^ { N } \sum _ { i , j = 1 } ^ { N _ { D } } \left(d_{i}^{(n)}\left(t+\tau_{\mathrm{d}}\right)\right.\right. \\
& \left.\left.-\left(\delta_{i j}+\alpha_{i j}\right) d_{j}^{(n)}(t)\right)^{2}\right]
\end{aligned}
$$




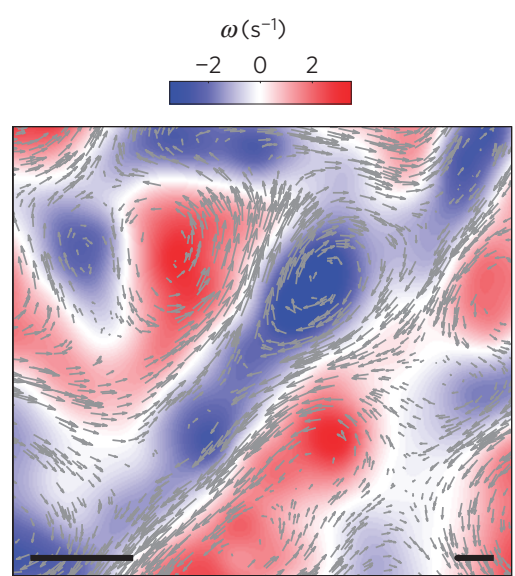

b

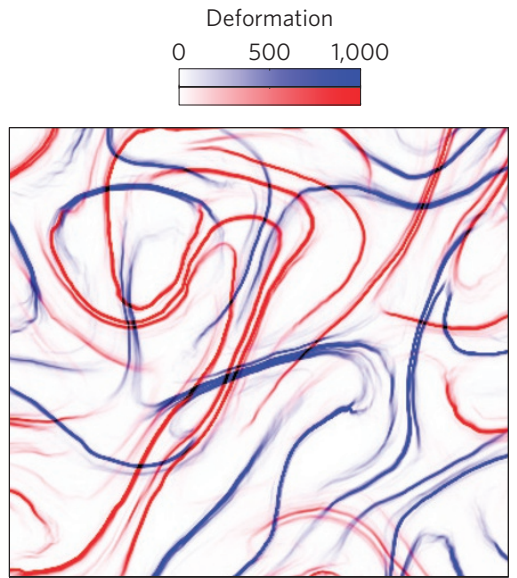

c
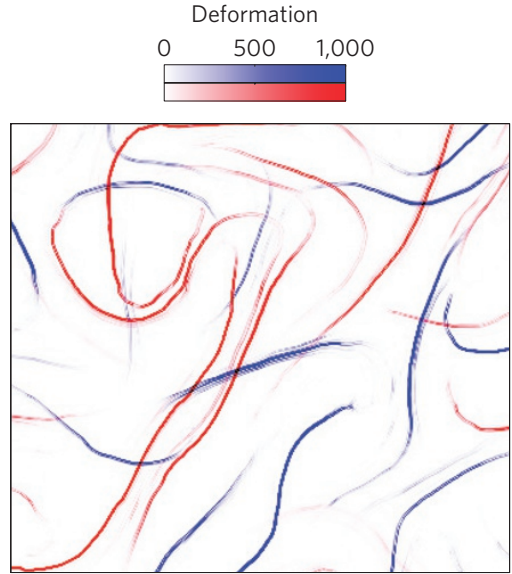

Figure 1 | Flow and deformation fields. a, Subregion of one frame of experimental data, with particle locations and velocities indicated by arrows and vorticity $\omega=\nabla \times \mathbf{u}$ (where $\mathbf{u}$ is the velocity) shown in colour. Here $R e=185 . \mathbf{b}$, Reverse-time (red) and forward-time (blue) linear, affine deformation $A^{2}$ in the same subregion, computed for $\left|\tau_{d}\right|=2 T_{L}=5.6 \mathrm{~s}$. c, Reverse-time (red) and forward-time (blue) nonlinear, non-affine deformation $D^{2}$. Both components of the deformation vary strongly with location. Left scale bar, dimensions of image $=2 \mathrm{~cm}$; right scale bar $=2 \mathrm{~cm} \mathrm{~s}^{-1}$, and represents the magnitude of the velocity indicated by arrows.

where all sums are explicit, $N_{D}=2$ is the space dimension, $\delta_{i j}$ is the Kronecker delta and $\alpha_{i j}$ is the best-fit (in a least-squares sense) affine deformation matrix ${ }^{11} . D^{2}$, the residual of this fit, is large when the linear model is poor, that is, when the deformation is far from affine. We can then express the total affine (stretching) component of the deformation as

$$
A^{2}\left(\mathbf{x}, t, \tau_{\mathrm{d}}\right)=\frac{1}{d_{0}^{2} N} \sum_{n=1}^{N} \sum_{i, j=1}^{N_{D}}\left(\alpha_{i j} d_{j}^{(n)}(t)\right)^{2}
$$

where $\alpha_{i j}$ is the same matrix that minimized $D^{2}$. Both $A^{2}$ and $D^{2}$ are non-dimensional. In our experiments, we used $N=12$ and $d_{0}=1.4 \mathrm{~mm}(\approx L / 18$, the typical distance between particles); the variation of our results with these two parameters is shown in Supplementary Figs S1 and S2. We note that, even though pure rotations are also affine, we have defined $A^{2}$ so that it measures primarily stretching in our flow.

By computing $A^{2}$ and $D^{2}$ at many $\mathbf{x}^{(0)}(t)$, we construct spatially resolved deformation fields, as shown in Fig. 1 for both positive and negative $\tau_{\mathrm{d}}$; that is, for the deformation that the fluid will experience (positive $\tau_{\mathrm{d}}$ ) and the deformation that it has experienced (negative $\left.\tau_{\mathrm{d}}\right)$. Both the affine and non-affine deformation vary strongly in space, suggesting that stretching and folding are highly nonuniform. Similar spatial variability has been demonstrated for flow nonlinearity ${ }^{26}$. Though these two deformation fields look similar, we show below that their behaviour as a function of $\tau_{\mathrm{d}}$ is quite different. Both fields resemble the stretching fields that have recently been used to identify so-called Lagrangian coherent structures ${ }^{18,27,28}$, which are deeply connected to mixing ${ }^{29}$. This result is unsurprising for the affine deformation, given that the stretching field is also a linearization of the total deformation, constructed by ignoring highorder spatial derivatives. It is intriguing, however, that the structure of the non-affine field is so similar. In particular, we note that the non-affine field, which shows regions where the evolution of the flow is highly nonlinear, is large only in a small subset of the total flow, even though the Reynolds number is both much larger than unity and larger than the critical value at which the flow becomes spatiotemporally chaotic $\left(\operatorname{Re} / \operatorname{Re}_{c} \sim 2.5\right)$. This result suggests that the Reynolds number alone is a poor measure of mixing efficiency.

The distinction between our deformation fields and Lagrangian coherent structures becomes clear by studying the behaviour of the deformation as a function of $\tau_{\mathrm{d}}$. Here, we focus only on
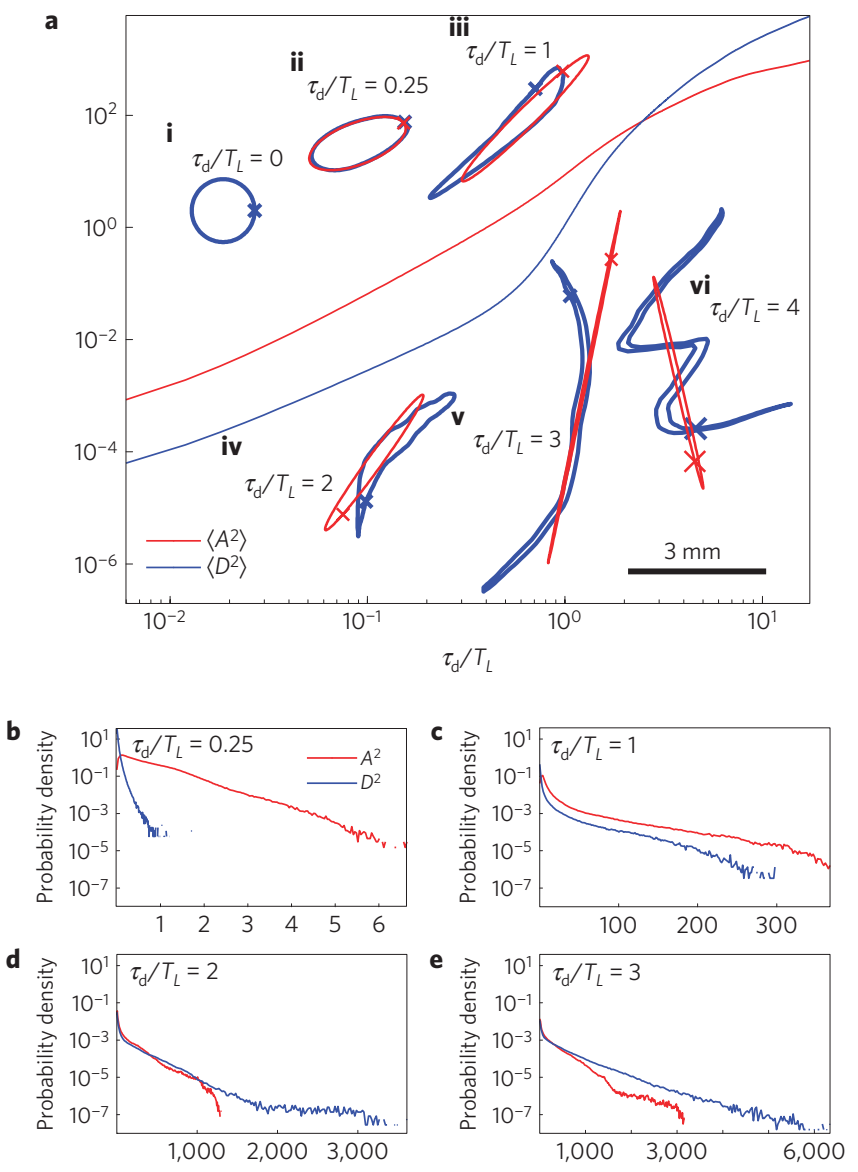

Figure 2 | Deformation varying with time. a, Average values of $A^{2}$ (red) and $D^{2}$ (blue) as a function of $\tau_{\mathrm{d}}$. The average of $D^{2}$ begins to increase rapidly once $\tau_{\mathrm{d}} \sim T_{L}$. Insets (i)-(vi), Progression of snapshots of the deformation of an initially circular material area in the same frame as in Fig. 1. The measured shape of the cluster is shown in blue, and the best-fit affine deformation is shown in red. One point is marked with an X to make rotations apparent, and the scale is constant. The indicated scale bar is roughly $1 \%$ of the camera's field of view. b-e, Probability density functions of $A^{2}$ (red) and $D^{2}$ (blue) for four different $\tau_{\mathrm{d}}$ values. The tail of the $D^{2}$ distribution grows rapidly once $\tau_{\mathrm{d}} \sim T_{L}$. 


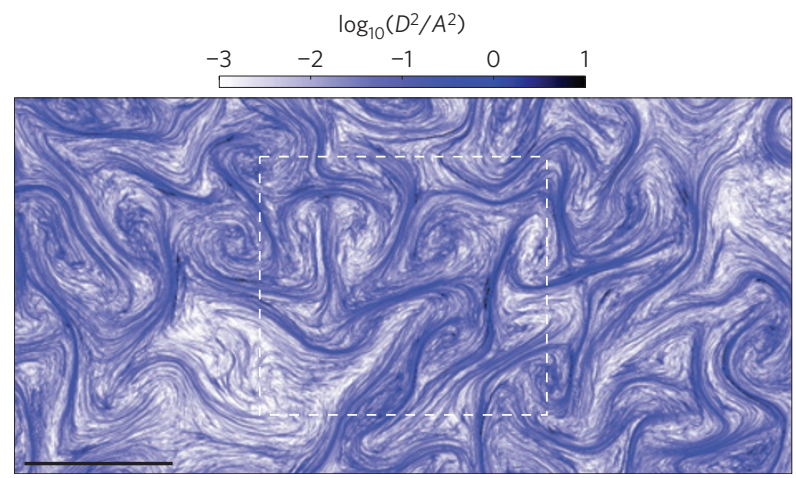

Figure 3 | Spatially resolved ratio of folding to stretching. The ratio of $D^{2}$ to $A^{2}$, plotted on a logarithmic colour scale to show detail, for the same data as shown in Fig. 1, with $\tau_{d}=2 T_{L}$. An outer margin of the measurement region is not shown so as to avoid spurious edge effects. The dashed central box indicates the subregion shown in Fig. 1. The relative importance of stretching and folding varies strongly with location. Scale bar, $5 \mathrm{~cm}$.

positive $\tau_{\mathrm{d}}$; our results for negative $\tau_{\mathrm{d}}$ are similar (as shown in Supplementary Fig. S3). By increasing $\tau_{\mathrm{d}}$ we can watch individual clusters deform over time; one such progression (chosen at random from our data set; other examples are shown in Supplementary Fig. S4) is shown in Fig. 2. This example suggests that deformation is a two-stage process: short-time deformation is dominated by stretching, whereas folding occurs at longer times. This hypothesis is borne out by measurements of $\left\langle A^{2}\right\rangle$ and $\left\langle D^{2}\right\rangle$ as a function of $\tau_{\mathrm{d}}$, where $\langle\cdot\rangle$ denotes an average over both space and $t$. As shown in Fig. $2 \mathrm{~g},\left\langle A^{2}\right\rangle$ far exceeds $\left\langle D^{2}\right\rangle$ at early times, but $\left\langle D^{2}\right\rangle$ increases suddenly at $\tau_{\mathrm{d}} \sim T_{L}$ and dominates $\left\langle A^{2}\right\rangle$ at later times. We also measured the mean Lagrangian coherent structure stretching field from our data; as shown in Supplementary Fig. S5, its average closely resembles $\left\langle A^{2}\right\rangle$ but is very different from $\left\langle D^{2}\right\rangle$. The full probability density functions of $A^{2}$ and $D^{2}$, shown in Fig. 2b-e, tell a similar story. Although the distributions of $A^{2}$ and $D^{2}$ both become broader as $\tau_{\mathrm{d}}$ increases, the tail of the $D^{2}$ distribution grows quickly for $\tau_{\mathrm{d}}>T_{L}$, and its width soon exceeds that of the $A^{2}$ distribution. The rapid change in the growth of the non-affine deformation at $\tau_{\mathrm{d}} \sim T_{L}$ coincides with the time when $A^{2} \sim 1$, corresponding to the doubling of the cluster's aspect ratio. This observation supports our hypothesis of a two-stage deformation process: a spherical volume cannot be folded without first being stretched. Although we show data from only one Re in Fig. 2, our results are similar for all other Re measured (as shown in Supplementary Fig. S6).

Although the averages of $A^{2}$ and $D^{2}$ in Fig. $2 \mathrm{~g}$ suggest that the relative importance of folding increases rapidly once $\tau_{\mathrm{d}} \sim T_{L}$, the heavy tails in the probability density functions imply that stretching and folding have significant spatial variability. In Fig. 3, we show the spatially resolved ratio of $D^{2}$ to $A^{2}$. This ratio is highly heterogeneous in space, varying by many orders of magnitude over very short length scales. Before $\tau_{\mathrm{d}} \sim T_{L}$, stretching exceeds folding nearly everywhere. Later, however, folding begins to dominate in isolated, line-like regions. These regions often lie close to the invariant manifolds of the hyperbolic critical points in the flow, as would be expected from a lobe-dynamics analysis ${ }^{30}$. To study the regions where $D^{2}>A^{2}$ in more detail, in Fig. 4a we plot the fraction of our measurement area where $D^{2}>A^{2}$ as a function of $\tau_{\mathrm{d}}$. Consistent with our earlier observations, stretching exceeds folding everywhere when the deformation time is short $\left(\tau_{\mathrm{d}} \ll T_{L}\right)$, but folding dominates nearly everywhere at later times $\left(\tau_{\mathrm{d}} \gg T_{L}\right)$. A progression of snapshots in Fig. $4 \mathrm{~b}-\mathrm{e}$ reveals the growth of the regions where $D^{2}>A^{2}$ as $\tau_{\mathrm{d}}$ increases, which proceeds quickly in the range $2 T_{L}<\tau_{\mathrm{d}}<4 T_{L}$. These regions are point-like at small $\tau_{\mathrm{d}}$, when the flow dynamics are well characterized by linearized equations.

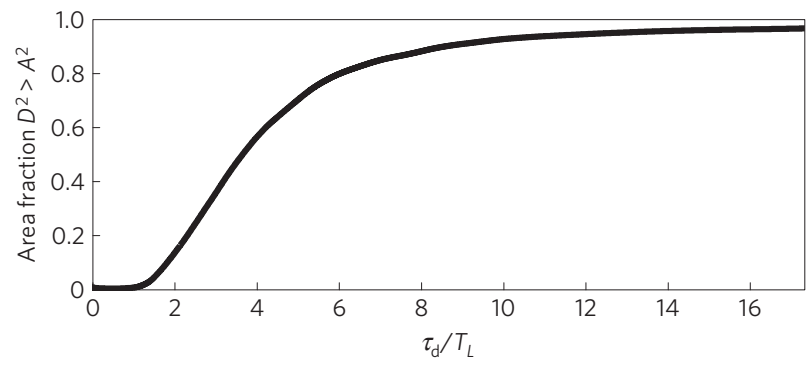

b

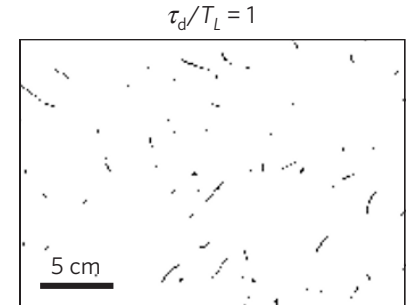

C $\tau_{\mathrm{d}} / T_{L}=2$

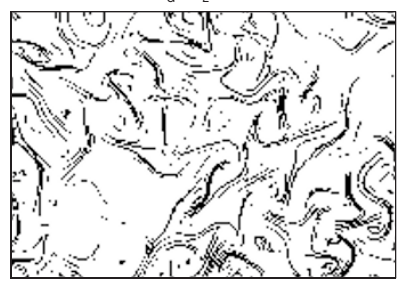

d $\tau_{\mathrm{d}} / T_{L}=3$

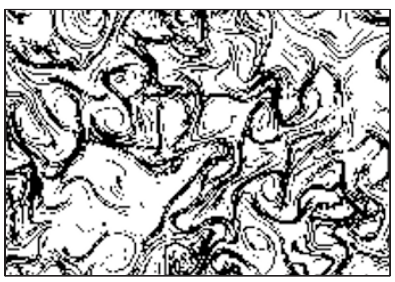

e $\tau_{\mathrm{d}} / T_{\mathrm{L}}=4$

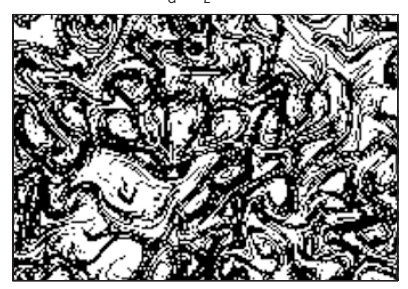

Figure 4 | Regions where folding dominates. a, The fraction of the measurement region where non-affine deformation exceeds affine deformation, for the same data as shown in Fig. 2. b-e, Regions where folding exceeds stretching, for the same data as shown in Figs 1 and 3 , at $\tau_{\mathrm{d}}=T_{L}, 2 T_{L}, 3 T_{L}$ and $4 T_{L}$. At short times, these regions are point-like. As $\tau_{\mathrm{d}}$ increases, they grow into lines that eventually join and become space filling at long times.

As $\tau_{\mathrm{d}}$ increases, however, these regions become line-like and finally space filling (with fractal dimension two): eventually, the entire flow undergoes strong nonlinear deformation.

Our experiments provide new insights in the details of fluid mixing. By explicitly considering the non-affine part of the fluid deformation, we can move past the linearized models commonly used to study this complex problem toward a fully nonlinear description. We have shown that stretching and folding operate on different timescales and that their relative strength depends strongly on space and time; similar effects are likely to be present in other situations described by stretching and folding, such as the evolution of magnetic field lines (and therefore magnetic energy density) in liquid metals and plasmas. Finally, we have shown that tools developed for studying glassy solids can be used to gain a deeper understanding of a fluid system, a result that suggests that further exchange of techniques between these communities may be fruitful.

Received 1 July 2010; accepted 1 February 2011; published online 6 March 2011

\section{References}

1. Jaeglé, L. Pumping up surface air. Science 315, 772-773 (2007).

2. Stroock, A. D. et al. Chaotic mixer for microchannels. Science 295, 647-651 (2002).

3. Stone, H. A., Stroock, A. D. \& Ajdari, A. Engineering flows in small devices: Microfluidics towards a lab-on-a-chip. Annu. Rev. Fluid Mech. 36, 381-411 (2004).

4. Vergassola, M., Villermaux, E. \& Shraiman, B. I. 'Infotaxis' as a strategy for searching without gradients. Nature 445, 406-409 (2007).

5. Aref, H. Stirring by chaotic advection. J. Fluid Mech. 143, 1-21 (1984).

6. Shraiman, B. I. \& Siggia, E. D. Scalar turbulence. Nature 405, 639-646 (2000). 
7. Reynolds, O. Study of fluid motion by means of coloured bands. Nature $\mathbf{5 0}$ 161-164 (1894).

8. Ottino, J. M. The Kinematics of Mixing: Stretching, Chaos, and Transport (Cambridge Univ. Press, 1989).

9. Ott, E. Chaos in Dynamical Systems (Cambridge Univ. Press, 2002).

10. Wiggins, S. Chaotic Transport in Dynamical Systems (Springer, 2010).

11. Falk, M. L. \& Langer, J. S. Dynamics of viscoplastic deformation in amorphous solids. Phys. Rev. E 57, 7192-7205 (1998).

12. Schall, P., Weitz, D. A. \& Spaepen, F. Structural rearrangements that govern flow in colloidal glasses. Science 318, 1895-1899 (2007).

13. Rothstein, D., Henry, E. \& Gollub, J. P. Persistent patterns in transient chaotic fluid mixing. Nature 401, 770-772 (1999).

14. Boyland, P. L., Aref, H. \& Stremler, M. A. Topological fluid mechanics of stirring. J. Fluid Mech. 403, 277-304 (2000).

15. Rogers, D. F. \& Adams, J. A. Mathematical Elements for Computer Graphics (McGraw-Hill, 1990).

16. Schuh, C. A. \& Lund, A. C. Atomistic basis for the plastic yield criterion of metallic glass. Nature Mater. 2, 449-452 (2003).

17. Lemaittre, A. Rearrangements and dilatancy for sheared dense materials. Phys. Rev. Lett. 89, 195503 (2002).

18. Voth, G. A., Haller, G. \& Gollub, J. P. Experimental measurements of stretching fields in fluid mixing. Phys. Rev. Lett. 88, 254501 (2002).

19. Rivera, M. K. \& Ecke, R. E. Pair dispersion and doubling time statistics in two-dimensional turbulence. Phys. Rev. Lett. 95, 194503 (2005).

20. Ouellette, N. T. \& Gollub, J. P. Curvature fields, topology, and the dynamics of spatiotemporal chaos. Phys. Rev. Lett. 99, 194502 (2007).

21. Ouellette, N. T., O'Malley, P. J. J. \& Gollub, J. P. Transport of finite-sized particles in chaotic flow. Phys. Rev. Lett. 101, 174504 (2008).

22. Ouellette, N. T., Xu, H. \& Bodenschatz, E. A quantitative study of three-dimensional Lagrangian particle tracking algorithms. Exp. Fluids 40, 301-313 (2006).

23. Vella, D. \& Mahadevan, L. The Cheerios effect. Am. J. Phys. 73, 817-825 (2005).

24. Lekien, F., Coulliette, C., Bank, R. \& Marsden, J. Open-boundary modal analysis: Interpolation, extrapolation, and filtering. J. Geophys. Res. 109, C12004 (2004).
25. Kelley, D. H. \& Ouellette, N. T. Direct multi-scale reconstruction of velocity fields from measurements of particle tracks. Preprint at http://arxiv.org/abs/1004.4687 (2010).

26. Egolf, D. A., Melnikov, I. V., Pesch, W. \& Ecke, R. E. Mechanisms of extensive spatiotemporal chaos in Rayleigh-Bénard convection. Nature 404, 733-736 (2000).

27. Haller, G. \& Yuan, G. Lagrangian coherent structures and mixing in two-dimensional turbulence. Physica D 147, 352-370 (2000).

28. Mathur, M., Haller, G., Peacock, T., Ruppert-Felsot, J. E. \& Swinney, H. L. Uncovering the Lagrangian skeleton of turbulence. Phys. Rev. Lett. 98, 144502 (2007).

29. Shadden, S. C., Lekien, F. \& Marsden, J. E. Definition and properties of Lagrangian coherent structures from finite-time Lyapunov exponents in two-dimensional aperiodic flows. Physica D 212, 271-304 (2005).

30. Malhotra, N. \& Wiggins, S. Geometric structures, lobe dynamics, and Lagrangian transport in flows with aperiodic time-dependence, with applications to Rossby wave flow. J. Nonlinear Sci. 8, 401-456 (1998).

\section{Acknowledgements}

This work was supported in part by the facilities and staff of the Yale University Faculty of Arts and Sciences High Performance Computing Center. We acknowledge financial support from the US National Science Foundation under grant No DMR-0906245.

\section{Author contributions}

N.T.O. conceived the original idea and supervised the project. D.H.K. ran the experiments and analysed the data. Both authors wrote the paper.

\section{Additional information}

The authors declare no competing financial interests. Supplementary information accompanies this paper on www.nature.com/naturephysics. Reprints and permissions information is available online at http://npg.nature.com/reprintsandpermissions. Correspondence and requests for materials should be addressed to N.T.O. 